\title{
Joint overbooking and seat allocation for fare families
}

\author{
Hjorth, Robert; Fiig, Thomas; Larsen, Jesper; Bondoux, Nicolas
}

Published in:

Journal of Revenue and Pricing Management

Link to article, DOI:

$10.1057 / \mathrm{s} 41272-018-0150-4$

Publication date:

2018

Document Version

Peer reviewed version

Link back to DTU Orbit

Citation (APA):

Hjorth, R., Fiig, T., Larsen, J., \& Bondoux, N. (2018). Joint overbooking and seat allocation for fare families. Journal of Revenue and Pricing Management, 17(6), 436-452. https://doi.org/10.1057/s41272-018-0150-4

\section{General rights}

Copyright and moral rights for the publications made accessible in the public portal are retained by the authors and/or other copyright owners and it is a condition of accessing publications that users recognise and abide by the legal requirements associated with these rights.

- Users may download and print one copy of any publication from the public portal for the purpose of private study or research.

- You may not further distribute the material or use it for any profit-making activity or commercial gain

- You may freely distribute the URL identifying the publication in the public portal 


\title{
Joint overbooking and seat allocation for fare families
}

\author{
Robert Hjorth \\ Beddingen 20,0250 Oslo, Norway. E-mail: hjorth.robert@gmail.com
}

Robert Hjorth is a Project Leader at The Boston Consulting Group (BCG). He holds an Elite MSc in Mathematical Modeling and Computation with specialization in Operations Research from the Technical University of Denmark (DTU). While parts of this research was conducted while Robert was a MSc student at DTU, the majority has been conducted independently.

\section{Thomas Fiig}

Amadeus Airline IT, Lufthavnsboulevarden 14, DK-2770 Kastrup, Denmark. E-mail: thomas.fig@sca.amadeus.com

Thomas Fiig is Director and Chief Scientist at Amadeus, where he is responsible for the revenue management strategy and scientific methodologies. He holds a PhD in Theoretical Physics and Mathematics and a BA in Finance from the University of Copenhagen, Denmark. Prior to joining Amadeus, he was Chief Scientist at Scandinavian Airlines System. He has published several articles, recently focused on methodologies for origin and destination forecasting and optimization of simplified fare structures. He serves on the editorial board of Journal of Revenue and Pricing Management.

\section{Jesper Larsen}

Technical University of Denmark, Department of Management Engineering, Produktionstorvet, DTU - Building 424, DK-2800 Kgs. Lyngby, Denmark. E-mail (corresponding author): jesla@dtu.dk

Jesper Larsen is Professor of Operations Research and Head of Management Science at the Department of Management Engineering at the Technical University of Denmark. He holds an MSc in Computer Science from the University of Copenhagen and a $\mathrm{PhD}$ in Operations Research from the Technical University of Denmark. Jesper has published several articles within transport optimization and scheduling.

\section{Nicolas Bondoux}

Amadeus Airline IT, Amadeus S.A.S., 485 Route du Pin Montard, 06902 Sophia Antipolis Cedex, France.E-mail: nbondoux@amadeus.com

Nicolas Bondoux is a research engineer at Amadeus, in the Analysis and Research department. Formerly being a contributor to Amadeus' revenue management products, his current main research interests are revenue management and artificial intelligence. He graduated from ENSIIE Engineering School in Évry, France and holds an MSc in Mathematics and Computer Science from the University of Manchester. 


\begin{abstract}
Revenue management systems (RMS) traditionally solve overbooking and seat allocation separately. Overbooking is managed by inflating the physical capacity using a static overbooking model, followed by seat allocation (excluding overbooking) on the inflated capacity. This simplification, although not optimal, has been the practice since the birth of revenue management. In this paper, we solve the joint overbooking and seat allocation problem for fare families by invoking the Equivalence Charging Transformation of Subramanian et al. [1999] and the Marginal Revenue Transformation of Fiig et al. [2010]. These transformations enable us to transform the high dimensional dynamic programming (DP) model into an equivalent low dimensional DP model that can readily be solved and implemented in existing RMS and inventory systems. We demonstrate that the DP model not only is able to incorporate factors that were previously ignored, such as the demand level, willingness to pay, and class-specific refund and cancellation rates, but importantly also provides significant revenue gains of 1 to 3 per cent at lower denied boarding levels compared with current industry practice.
\end{abstract}

Keywords: Revenue management systems (RMS); seat allocation; overbooking; fare families; dynamic programming (DP)

\title{
1 Introduction and Motivation
}

Smith et al. [1992] estimate that 15 per cent of seats on American Airlines would have been spoiled on sold-out flights without overbooking. This agrees well with Gorin et al. [2006], who recently estimated that 15 to 18 per cent of the total revenue stems from overbooking based on data from Continental Airlines. This shows that overbooking provides tremendous value to the airlines. However, overbooking also creates denied boardings (DBs), which can be a painful experience - as witnessed by United Airlines' controversy and public relations disaster after having a passenger dragged off the plane, resulting in the CEO publicly apologizing and initiating policy changes to improve the service, incl. offering (up to) $\$ 10,000$ for a voluntary DB (see e.g. LeBeau [2017]).

Therefore, it is of great commercial interest to develop optimization methodologies that allow for improving the total airline revenue while at the same time lowering the DB level.

To our knowledge, in all commercial revenue management systems (RMS), overbooking and seat allocation problems are solved separately. Overbooking is managed by inflating the physical capacity using a static overbooking model (variation on the model of Rothstein and Stone [1967]), followed by seat allocation (excluding overbooking) on the inflated capacity. RMS was constructed this way out of necessity, because computational complexity prohibited solving the correct optimization problem.

The traditional RMS was built assuming a business environment of strong product differentiation (so-called fenced fare structures), yet the business environment has changed in response to competition from low-cost carriers (LCCs) in particular. Today, we see an evolution of the fare structure from fenced fare structures into fare families. Note therefore the many ways in which current overbooking practice is inadequate:

- Addressing overbooking separately from seat allocation ignores their relationship.

- It is inconsistent with the underlying fare structure, which has evolved from fenced fare structures (often limited to one fare product) into fare families.

- Overbooking models ignore important factors that affect the overbooking level, such as demand level, class mix, and class-specific refund and cancellation rates.

Our objective is to solve the joint overbooking and seat allocation problem for fare families. To this end, we select as starting point the $m$-dimensional (abbreviated $m$-dim, where $m$ is the number of fare classes) DP model for joint overbooking and seat allocation of a general fare structure, which was independently formulated by Hjorth [2013] and Sierag et al. [2015]. In order to solve this model, we apply the mathematical tools: The Equivalence Charging Transformation (ECT) of Subramanian et al. [1999] and the Marginal Revenue Transformation (MRT) of Fiig et al. [2010]. 
The ECT transformation allows us to transform the original $m$-dim DP problem into an equivalent $n$-dim DP problem ( $n$ being the number of fare families). This already makes good progress as typically $n \leq 3$ while $m=26$. However, while it may be computationally feasible to solve the $n$-dim DP problem, it cannot be generalized to a network with multiple traffic flows across a leg. This requires that the DP model can be formulated as an independent demand DP model (in order to prioritize among the flows). The MRT fulfils exactly this purpose, which, given some assumptions on the cancellation rates, enables us to transform the $n$-dim DP problem to a 1-dim DP problem.

Finally, we benchmark the exact $n$-dim DP model and 1-dim DP model against current industry practice. We demonstrate that the 1-dim DP model provides near-optimal performance on all measured key performance indicators. Further, the 1-dim DP model overcomes the inadequacies mentioned above and delivers significant revenue gains with fewer DBs.

We believe that our contribution is of substantial commercial value, given that we demonstrate that our 1-dim DP model for fare families not only is able to incorporate factors that were previously ignored, such as the demand level, willingness to pay (WTP), and class-specific refund and cancellation rates, but importantly also provides significant revenue gains at lower DB levels compared with current industry practice. Further, the DP model can readily be implemented in a network environment using existing RMS and inventory systems.

The structure of our paper is as follows: In Section 2, we provide a comprehensive literature review. In Section 3, we establish equivalence between selected DP models by means of the ECT and MRT. In Section 4, we apply the ECT to the dependent demand DP model and extend the MRT to include the cost of cancellations. We apply this methodology to fare families and demonstrate how the resulting $n$-dim DP model may be reduced to a 1-dim DP model under certain assumptions. In Section 5, we benchmark the exact $n$-dim DP model and 1-dim DP model against current industry practice. Finally, we draw our conclusions in Section 6.

\section{Literature Review}

The complete history of overbooking models since Rothstein and Stone [1967] is too lengthy and beyond the scope to repeat here. Review papers by Rothstein [1985] and Ratliff [1998] cover this history in a concise and readable way.

The model evolution over the last few decades, as they relate to single-leg seat allocation and overbooking, can be organized according to two axes: Type of optimization model (pure seat allocation, pure overbooking, and joint overbooking and seat allocation) and Type of demand model (independent demand [appropriate for fenced fare structures] or dependent demand [appropriate for simplified or fare family fare structures]).

The seat allocation models are often divided into static and dynamic models. In static models, we assume that multiple fare products book in a sequential order, typically from low to high fares as the departure date approaches. In DP models, no assumptions are made about the booking order among fare products. Initially, the seat allocation problem was treated using static models in Littlewood [2005] and Belobaba [1989], while, later, DP models were applied in Lee and Hersh [1993] and Lautenbacher and Stidham [1999].

The traditional RMS was built assuming a business environment of strong product differentiation (so-called fenced fare structures), which enabled a critical simplification: Demand independence by fare product. This assumption was the foundation of the 1980s- and 1990s-era RMS that are still prevalent today, yet the business environment has changed in response to competition from incumbent airlines and LCCs.

In the 2000s, simplified fare structures started to emerge, primarily driven by competition from LCCs. The simplified fare structures invalidated the independent demand assumption, causing the RMS to spiral down to the lowest fare class and leading to tremendous loss of revenue (see Cooper et al. [2006]).

The theoretical resolution was published in a seminal paper by Talluri and van Ryzin [2004a], that introduced customer choice behavior into a DP model, thereby explicitly accounting for the 
demand dependence between classes. The MRT by Fiig et al. [2010] made the model implementable in practice by transforming it into an equivalent independent demand model (with adjusted fares and demands), thereby allowing for application to real airline networks.

Similarly, we divide overbooking models into static and dynamic models. In static models, the booking horizon is split into two stages: A booking period, followed by a service period (following the terminology of Talluri and van Ryzin [2004b]). In the static models, typically, only one fare is considered, chosen to represent the average fare (so-called spoilage cost).

The DP models allow for more realism by considering booking events as well, and, in the case of joint overbooking and seat allocation models, multiple fare products. The downside is the intractability of these models.

Rothstein and Stone [1967] were among the first to model overbooking using a static model. Later, Shlifer and Vardi [1975] extended the work of Rothstein and Stone [1967] to the following cases: (i) The single-leg model with two types of passengers with different cancellation rates, and (ii) A twoleg flight with three segments (two local and a connection), each with different cancellation rates. However, even these simple generalizations create complex accept/reject regions of the inventory state space, making the models of limited practical applicability.

Bodily and Pfeifer [1992] relaxed the assumption of a constant survival rate of the Rothstein and Stone [1967] model to allow time-to-departure-dependent survival rates. The resulting decision rule is provided below for later reference. Consider a single-leg flight with capacity $C$, and denote the optimal overbooking level $O B_{t}$ (gross capacity is $\left[C+O B_{t}\right]$ ). Let the cancellation rate at time $t$ be denoted $q_{t}$ and the denied boarding compensation and spoilage cost be denoted $D B C$ and $f$ respectively. Then, the optimal overbooking level can be determined by

$$
\frac{C-\left(C+O B_{t}\right)\left(1-q_{t}\right)}{\sqrt{\left(C+O B_{t}\right)\left(1-q_{t}\right) q_{t}}}=\Phi^{-1}\left(\frac{D B C}{f+D B C}\right)
$$

where $\Phi^{-1}$ is the inverse normal distribution function. This expression allows for an analytical solution of the overbooking level.

Klophaus and Pölt [2007] extended the Bodily and Pfeifer [1992] model to allow dynamic spoilage cost $f$ and hence better represent customer's WTP across the booking horizon. Simulation studies on real Lufthansa data displayed minor incremental revenue gains over a static spoilage cost.

Rothstein [1971] was the first to formulate a dynamic overbooking model. He formulated it as a non-homogenous Markov process, and solved the model using DP. However, in practice (see Talluri and van Ryzin [2004b]) static models are often preferred to DP models due to their simplicity and robustness. For this reason, Wang and Walczak [2016] recently compared a variety of static and dynamic overbooking models. They concluded that the DP models, while sharing many of the characteristics of static models, consistently outperform the static models in terms of revenue and with fewer DBs.

The joint overbooking and seat allocation problem was first considered by Alstrup and Boas [1984], who formulated a 2-dim DP model for two cabins, while accounting for complexities introduced by actual aircraft configurations, such as the position of the cabin divider and upgrades and downgrades. Later, Subramanian et al. [1999] extended the results of Lee and Hersh [1993] for a single fare class problem to a single-cabin flight with multiple fare classes, and included cancellations, no-shows, and overbooking. They demonstrated under realistic conditions that a booking limit control mechanism is optimal.

Recently, Hjorth [2013] and Sierag et al. [2015] independently combined the models of Talluri and van Ryzin [2004a] and Subramanian et al. [1999], thereby generalizing the above models for joint overbooking and seat allocation to include customer choice behavior in a general fare structure. While the general formulation is intractable due to the curse of dimensionality, heuristic models that allow for (accurate) approximate solutions can be obtained under certain conditions. The present paper can be seen as developing such models for the special case of fare families. 


\section{Overview of Published DP Models and Transformations}

In this section, we show how the DP models of Lee and Hersh [1993], Subramanian et al. [1999], Talluri and van Ryzin [2004a], Hjorth [2013] and Sierag et al. [2015] are linked together, and in fact can be derived from each other through application of the transformations of Subramanian et al. [1999] and Fiig et al. [2010]. The relationship between the models, transformations, and present work, is depicted in Figure 1. The models are grouped according to the same axes as employed in Section 2: Type of optimization model and Type of demand model. We show the dimensionality of the state variable and the acceptance criteria for each model. An arrow represents exact equivalence between the models.

For clarity, we name the models according to types of demand model (dependent [D] vs. independent $[\mathrm{I}]$ ), optimization model (separate vs. joint overbooking and seat allocation [OB]), and how cancellation cost is treated (total cost [TC] of cancellations when refund is paid at the time of cancellation vs. expected unit cost $[\mathrm{UC}]$ of cancellation at time of booking when refund is considered up-front). We will assess the existing DP models in chronological order.

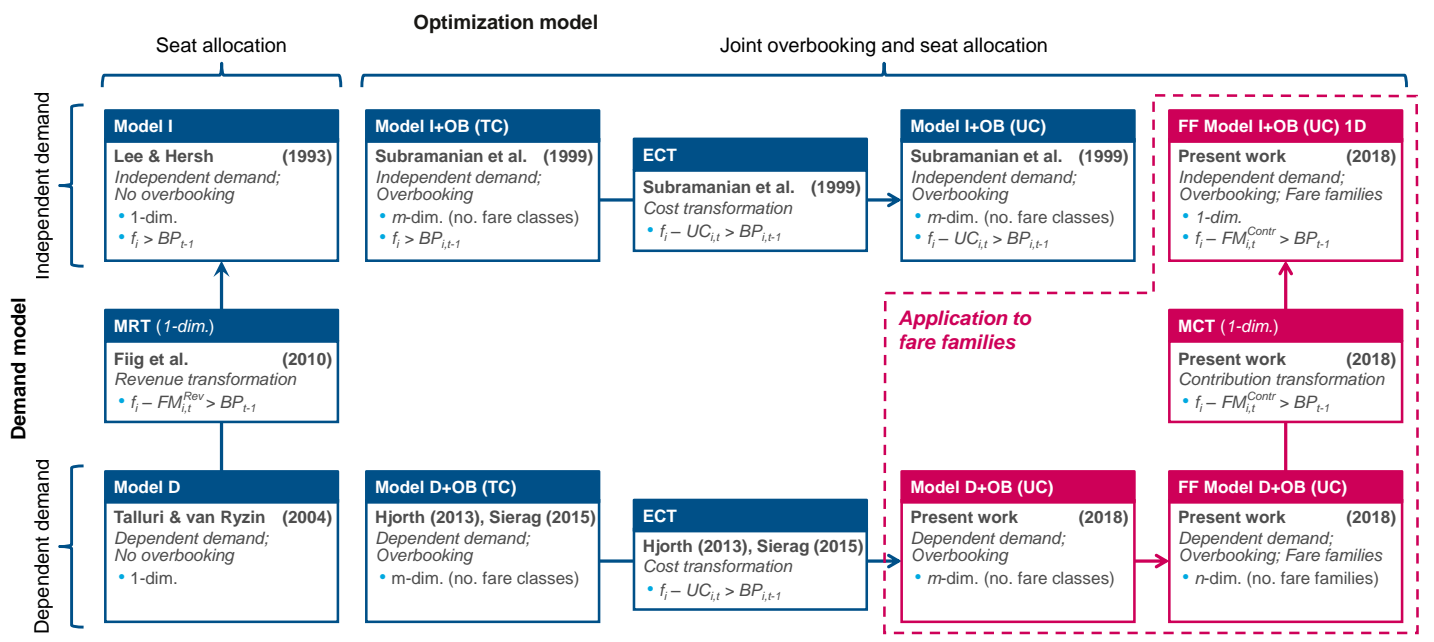

Figure 1: Relations between DP models, established through ECT and MRT. These relations enable the solution of the joint overbooking and seat allocation model for fare families. Our simulations (see Section 5) are performed by use of FF Model D+OB (UC) ( $n$-dim exact model) and FF Model I+OB (UC) 1D (1-dim approximate model). The comparison baseline model (not shown) is a hybrid model with overbooking performed by (1) and seat allocation by Model I.

\section{Notation for General Fare Structures}

There are $m$ fare classes, and we employ either an $m$-dim state variable $\mathbf{x}=\left(x_{1}, \ldots, x_{m}\right)$ or a 1-dim state variable with $x=\sum_{i=1}^{m} x_{i}$ for the pure seat allocation models. Fares are denoted by $f_{i}$ for fare class $i$ and are listed in decreasing fare order: $f_{i}>f_{i+1}, i=1, \ldots, m-1$. Notation is adapted from Subramanian et al. [1999]: 


\begin{tabular}{|c|c|}
\hline$C$ & hysical capacity \\
\hline$O B_{t}$ & Optimal overbooking level \\
\hline$T+1$ & Time periods, numbered in reverse order with departure at $t=0$ \\
\hline$d_{i, p, t}$ & $\begin{array}{l}\text { Demand for fare class } i \text { given that policy } p \text { is offered in time period } t \text { (policy } \\
\text { index removed if only one fare family) }\end{array}$ \\
\hline$D_{p, t}$ & Aggregated demand for policy $p$ in time period $t$, equal to $\sum_{i \in p} d_{i, p, t}$ \\
\hline$q_{i, t}$ & Cancellation rate for fare class $i$ in time period $t$ \\
\hline$c_{i}$ & $\begin{array}{l}\text { efund rate for fare class } i \text {, where we omit the time index } t \text { for the sake of } \\
\text { otational simplicity }\end{array}$ \\
\hline$\pi(\cdot)$ & Overbooking penalty function \\
\hline$T N_{t}(\mathbf{x})$ & Expected remaining net revenue in state $\mathbf{x}$ from time period $t$ to departure \\
\hline$T C_{t}(\mathbf{x})$ & xpected cost of cancellation in state $\mathbf{x}$ from time period $t$ to departure \\
\hline$T R_{t}(\mathbf{x})$ & $\begin{array}{l}\text { Gross total revenue in state } \mathbf{x} \text { from time period } t \text { to departure, defined by } \\
T N_{t}(\mathbf{x})+T C_{t}(\mathbf{x})\end{array}$ \\
\hline$U C$ & $\begin{array}{l}\text { Expected unit cost of cancellation in state } \mathbf{x} \text {, time period } t \text { attributable to } \\
\text { one incremental fare class } i \text { booking, defined by } T C_{i, t-1}\left(x_{i}+1\right)-T C_{i, t-1}\left(x_{i}\right)\end{array}$ \\
\hline$B P_{i, t-1}(\mathbf{x})$ & $\begin{array}{l}i \text { 'th component of the bid-price vector, equal to } T N_{t-1}(\mathbf{x})-T N_{t-1}\left(\mathbf{x}+\mathbf{e}_{i}\right) \\
\text { or } T R_{t-1}(\mathbf{x})-T R_{t-1}\left(\mathbf{x}+\mathbf{e}_{i}\right) \text { where } \mathbf{e}_{i} \text { is the } i \text { 'th unit vector }\end{array}$ \\
\hline$F M_{i, t}^{R e v}$ & Fare modifier adjusting revenue for fare class $i$ in time period $t$ \\
\hline & Fare modifier adjusting contribution of fare class $i$ in time period $t$ \\
\hline
\end{tabular}

\section{Model I: Lee and Hersh [1993]}

Assumptions: Seat allocation DP model with 1-dim state variable, demand independence by fare product, and no cancellations or overbooking.

Lee and Hersh [1993] were the first to consider seat allocation without overbooking by applying DP. We have chosen to use the simpler, but equivalent, formulation of Lautenbacher and Stidham [1999] for Model I

$$
T N_{t}(x)=\sum_{i=1}^{m} d_{i, t} \times \max \left\{f_{i}-B P_{t-1}(x), 0\right\}+T N_{t-1}(x)
$$

in which a booking is accepted if $f_{i}-B P_{t-1}(x)>0$ and rejected otherwise. The boundary conditions are $T N_{t}(C)=0$ (no further obtainable revenue when capacity is reached) and $T N_{0}(x)=0$ (no further revenue at time of departure). Lee and Hersh [1993] proved that the BP is non-decreasing in $x$ for constant $t$, i.e. $B P_{t}(x) \leq B P_{t}(x+1)$ (inventory monotonicity), and non-decreasing in $t$ for constant $x$, i.e. $B P_{t-1}(x) \leq B P_{t}(x)$ (time monotonicity). These properties guarantee existence of an optimal booking limit for each fare class $i$ in each time period $t$. Likewise, they guarantee existence of an optimal time for when to close each fare class $i$ for a given state $x$.

\section{Model I+OB (TC): Subramanian et al. [1999]}

Assumptions: Joint overbooking and seat allocation DP model with $m$-dim state variable, demand independence by fare product, cancellations and overbooking, and refund considered at time of cancellation.

Subramanian et al. [1999] extended Model I to include cancellations, no-shows, refund, and overbooking (in this paper, we exclude no-shows for the sake of notational simplicity but without loss of generality). The recursion equation for Model I+OB (TC) becomes

$$
\begin{array}{r}
T N_{t}(\mathbf{x})=\sum_{i=1}^{m} d_{i, t} \times \max \left\{f_{i}-B P_{i, t-1}(\mathbf{x}), 0\right\}+\sum_{i=1}^{m} q_{i, t} x_{i}\left(-c_{i}+T N_{t-1}\left(\mathbf{x}-\mathbf{e}_{i}\right)\right)+ \\
\left(1-\sum_{i=1}^{m} q_{i, t} x_{i}\right) T N_{t-1}(\mathbf{x})
\end{array}
$$


We will assume (as also done in Subramanian et al. [1999]) that (a) cancellations in one fare class are independent of the number of bookings in other fare classes, and (b) that cancellations are independent of each other, so the expected number of cancellations in fare class $i$ in time period $t$ is $q_{i, t} x_{i}$. Because overbooking is allowed, DBs also have to be considered. The boundary condition is $T N_{0}(\mathbf{x})=-\pi(\mathbf{x})$, where $\pi(\cdot)$ is a convex and non-decreasing penalty function expressing the cost of DBs. In the general case of Model I+OB (TC) with $m$ fare classes with different cancellation rates, the BP satisfies inventory monotonicity but not time monotonicity.

\section{Equivalence Charging Transformation: Subramanian et al. [1999]}

To overcome the computational complexity of having $m$ dimensions, Subramanian et al. [1999] developed a clever method to reformulate the problem. In Model I+OB (TC), the cancellation cost is treated when the cancellation event occurs. The ECT transforms the $m$-dim recursion equation into an equivalent set of equations in which the expected cancellation cost is instead assessed at the time of booking. $T C_{t}(\mathbf{x})$ is given by the recursion equation

$$
T C_{t}(\mathbf{x})=\sum_{i=1}^{m} q_{i, t} x_{i}\left(c_{i}+T C_{t-1}\left(\mathbf{x}-\mathbf{e}_{i}\right)\right)+\left(1-\sum_{i=1}^{m} q_{i, t} x_{i}\right) T C_{t-1}(\mathbf{x})
$$

where $T C_{0}(\mathbf{x})=0$. Note that $T C_{t}(\mathbf{x})$ is positive by convention. By using the definition of $T R_{t}(\mathbf{x})$ and inserting $T R_{t}(\mathbf{x})-T C_{t}(\mathbf{x})$ in place of $T N_{t}(\mathbf{x})$ in (3) we obtain

$$
\begin{aligned}
T R_{t}(\mathbf{x}) & =\sum_{i=1}^{m} d_{i, t} \times \max \left\{f_{i}-\left[T C_{t-1}\left(\mathbf{x}+\mathbf{e}_{i}\right)-T C_{t-1}(\mathbf{x})\right]-B P_{i, t-1}(\mathbf{x}), 0\right\} \\
& +\sum_{i=1}^{m} q_{i, t} x_{i} T R_{t-1}\left(\mathbf{x}-\mathbf{e}_{i}\right)+\left(1-\sum_{i=1}^{m} q_{i, t} x_{i}\right) T R_{t-1}(\mathbf{x})
\end{aligned}
$$

where $B P_{i, t-1}(\mathbf{x})=T R_{t-1}(\mathbf{x})-T R_{t-1}\left(\mathbf{x}+\mathbf{e}_{i}\right)$. Applying assumption (a) yields $T C_{t}(\mathbf{x})=\sum_{i=1}^{m} T C_{i, t}\left(x_{i}\right)$, where

$$
T C_{i, t}\left(x_{i}\right)=q_{i, t} x_{i}\left(c_{i}+T C_{i, t-1}\left(x_{i}-1\right)\right)+\left(1-q_{i, t} x_{i}\right) T C_{i, t-1}\left(x_{i}\right)
$$

with $T C_{i, 0}\left(x_{i}\right)=0$ for all $i$. From this 1-dim recursion equation we can define the expected unit cost of cancellation attributable to one incremental booking in fare class $i$ as $U C_{i, t}\left(x_{i}\right)=T C_{i, t-1}\left(x_{i}+\right.$ 1) $-T C_{i, t-1}\left(x_{i}\right)$. Assumption (b) implies that the unit cost is independent of $x_{i}$, and that $U C_{i, t}$ can be determined by the recursion equation

$$
U C_{i, t}=q_{i, t-1} c_{i}+\left(1-q_{i, t-1}\right) U C_{i, t-1}
$$

for $t \geq 2$ and with $U C_{i, 1}=0$. Finally, we replace each component of the vector $T C_{t-1}\left(\mathbf{x}+\mathbf{e}_{i}\right)-$ $T C_{t-1}(\mathbf{x})$ in (5) with the unit cost $U C_{i, t}$ to obtain Model I+OB (UC).

\section{Model I+OB (UC): Subramanian et al. [1999]}

Assumptions: Joint overbooking and seat allocation DP model with $m$-dim state variable, demand independence by fare product, cancellations and overbooking, and refund considered at time of booking.

Model I+OB (UC) and Model I+OB (TC) are equivalent, the only difference being how cancellation cost is treated; at the time of booking rather than at the time of cancellation. The recursion equation for Model $\mathrm{I}+\mathrm{OB}(\mathrm{UC})$, as derived above, is

$$
\begin{array}{r}
T R_{t}(\mathbf{x})=\sum_{i=1}^{m} d_{i, t} \times \max \left\{f_{i}-U C_{i, t}-B P_{i, t-1}(\mathbf{x}), 0\right\} \\
+\sum_{i=1}^{m} q_{i, t} x_{i} T R_{t-1}\left(\mathbf{x}-\mathbf{e}_{i}\right)+ \\
\left(1-\sum_{i=1}^{m} q_{i, t} x_{i}\right) T R_{t-1}(\mathbf{x})
\end{array}
$$


where $B P_{i, t-1}(\mathbf{x})=T R_{t-1}(\mathbf{x})-T R_{t-1}\left(\mathbf{x}+\mathbf{e}_{i}\right)$. DBs are considered through the boundary condition $T R_{0}(\mathbf{x})=-\pi(\mathbf{x})$. The ECT preserves monotonicity properties, so the BP in Model I+OB (UC) still satisfies inventory monotonicity but not time monotonicity.

\section{Model D: Talluri and van Ryzin [2004a]}

Assumptions: Seat allocation DP model with 1-dim state variable, demand dependence by fare product, and no cancellations or overbooking.

Talluri and van Ryzin [2004a] formulated a DP model in which demand depends on the policy offered and consumer behavior is expressed as a general discrete choice model. The control problem is different from the independent demand problem given that the DP model has to select the policy (among all policies) that maximizes $T N_{t}(x)$. The recursion equation for Model $\mathrm{D}$ is

$$
T N_{t}(x)=\max _{p}\left\{\sum_{i \in p} d_{i, p, t}\left(f_{i}-B P_{t-1}(x)\right\}+T N_{t-1}(x)\right.
$$

where $B P_{t-1}(x)=T N_{t-1}(x)-T N_{t-1}(x+1)$. Similar to Model I, the boundary conditions are $T N_{t}(C)=0$ and $T N_{0}(x)=0$ because cancellations and overbooking are not considered. Talluri and van Ryzin [2004a] proved identical monotonicity properties for Model D as Lee and Hersh [1993] did for Model I.

\section{Marginal Revenue Transformation: Fiig et al. [2010]}

Fiig et al. [2010] demonstrated how to transform a dependent demand model into an equivalent independent demand model by means of the MRT. In the dependent model, optimization is performed over all policies, and revenue and demand depend on the policy being offered. In time period $t$, aggregated demand for policy $p$ is $D_{p, t}=\sum_{i \in p} d_{i, p, t}$ and revenue is $T R_{p, t}=\sum_{i \in p} d_{i, p, t} f_{i}$ (note the notational distinction between $T R_{p, t}$, which by convention denotes the revenue within time period $t$, and $T R_{t}(x)$, which denotes the remaining revenue from time period $t$ to departure). In order to find the optimal policy, we can plot $D_{p, t}, T R_{p, t}$ in a scatterplot for all policies. This will trace out an efficient frontier.

The central idea of Fiig et al. [2010] is to create an equivalent efficient frontier consisting of transformed fare classes with independent demand. The transformed problem will have an equivalent solution due to the efficient frontiers being equal. This is achieved by a two-step approach. The first step is to create a virtual policy with independent demand for each efficient policy equal to the marginal demand $M D_{p, t}$ obtained by opening the next policy with dependent demand on the efficient frontier, and adjusted fare equal to the marginal revenue $M R_{p, t}$. The marginal demand and marginal revenue of opening policy $p$ instead of $p-1$ are

$$
\begin{aligned}
M D_{p, t} & =D_{p, t}-D_{p-1, t} \\
M R_{p, t} & =\left(T R_{p, t}-T R_{p-1, t}\right) /\left(D_{p, t}-D_{p-1, t}\right)
\end{aligned}
$$

where $p=1, \ldots, k$ denotes the $k$ efficient policies. By construction, the policies trace out an efficient frontier equivalent to the one traced out by the dependent demand policies.

The second step of the MRT is to map the policies back to the $m$ fare classes. As noted in Fiig et al. [2010], this is possible only if the policies along the efficient frontier are nested. In that case, the policies can be mapped to fare classes by assigning to each fare class opening for the first time $M R_{i, t}=f_{i}-F M_{i, t}^{R e v}$ as an adjusted fare and $M D_{i, t}$ as demand, where we have introduced the fare modifier $F M_{i, t}^{R e v}$ as the resulting fare adjustment for fare class $i$. The MRT demonstrates equivalence between Model D and Model I. 


\section{Model D+OB (TC): Hjorth [2013] and Sierag et al. [2015]}

Assumptions: Joint overbooking and seat allocation DP model with m-dim state variable, demand dependence by fare product, cancellations and overbooking, and refund considered at time of cancellation.

Hjorth [2013] and Sierag et al. [2015] combined the models of Talluri and van Ryzin [2004a] and Subramanian et al. [1999], thereby generalizing the above models for joint overbooking and seat allocation to include customer choice behavior in a general fare structure. The recursion equation of Model $\mathrm{D}+\mathrm{OB}(\mathrm{TC})$ is

$$
\begin{array}{r}
T N_{t}(\mathbf{x})=\max _{p}\left\{\sum_{i \in p} d_{i, p, t}\left(f_{i}-B P_{i, t-1}(\mathbf{x})\right)\right\}+\sum_{i=1}^{m} q_{i, t} x_{i}\left(-c_{i}+T N_{t-1}\left(\mathbf{x}-\mathbf{e}_{i}\right)\right)+ \\
\left(1-\sum_{i=1}^{m} q_{i, t} x_{i}\right) T N_{t-1}(\mathbf{x})
\end{array}
$$

where $B P_{i, t-1}(\mathbf{x})=T N_{t-1}(\mathbf{x})-T N_{t-1}\left(\mathbf{x}+\mathbf{e}_{i}\right)$ and the boundary condition $T N_{0}(\mathbf{x})=-\pi(\mathbf{x})$ is the overbooking penalty function.

\section{Joint Overbooking and Seat Allocation for Fare Families: Present Work [2018]}

The previous section covered a selection of published DP models and transformations (marked in blue in Figure 1). In this section, we complement the existing theory by extending the MRT to a Marginal Contribution Transformation (MCT) and then apply it to fare families (marked in red in Figure 1).

We first invoke the ECT on Model D+OB (TC) to derive Model D+OB (UC), and subsequently invoke the MCT to demonstrate equivalence between Model D+OB (TC) and Model I+OB (UC) under a simplifying assumption on the cancellation rates. We then apply Model D+OB (TC) to a fare family fare structure and invoke the ECT to rewrite the $m$-dim problem as the equivalent $n$-dim FF Model D+OB (UC) ( $n$ being the number of fare families). By making realistic assumptions on the cancellation rates, we invoke the MCT to transform the $n$-dim DP problem into FF Model $\mathrm{I}+\mathrm{OB}(\mathrm{UC})$ 1D. Interestingly, this model has the same structural form as Model I+OB (UC) that was derived for a single fare class.

\section{Notation for Fare Families}

We use the previous notation but add an index $j$ to denote the $n$ fare families. We employ an $n$-dim state variable $\mathbf{x}=\left(x_{1}, \ldots, x_{n}\right)$ or a 1 -dim state variable with $x=\sum_{i=1}^{m} x_{i}$. Let $f_{j, p}=\min _{i \in j, i \in p}\left\{f_{i}\right\}$ denote the lowest offered fare in fare family $j$, policy $p$, and adopt the following notation:

$$
\begin{array}{ll}
D_{j, p, t} & \text { Aggregated demand for fare family } j \text { given that policy } p \text { is offered in time } \\
& \text { period } t, \text { equal to } \sum_{i \in j} d_{i, p, t} \\
& \text { Cancellation rate for all fare classes in fare family } j \text { in time period } t \\
q_{j, t} & \text { Expected unit cost of cancellation in time period } t \text { attributable to one in- } \\
U C_{j, p, t} & \text { cremental booking in the lowest offered fare class in fare family } j, \text { policy } \\
& p
\end{array}
$$

\section{Model D+OB (UC)}

Assumptions: Joint overbooking and seat allocation DP model with $m$-dim state variable, demand dependence by fare product, cancellations and overbooking, and refund considered at time of booking. 
Starting with the recursion equation of Model D+OB (TC) as defined in (10), we invoke the ECT by calculating the total revenue of operating the system from $t$ to departure as $T R_{t}(\mathbf{x})=$ $T N_{t}(\mathbf{x})+T C_{t}(\mathbf{x})$ and inserting the total contribution $T R_{t}(\mathbf{x})-T C_{t}(\mathbf{x})$ in place of $T N_{t}(\mathbf{x})$ in (10) to obtain

$$
\begin{aligned}
T R_{t}(\mathbf{x}) & =\max _{p}\left\{\sum_{i \in p} d_{i, p, t}\left(f_{i}-\left[T C_{t-1}\left(\mathbf{x}+\mathbf{e}_{i}\right)-T C_{t-1}(\mathbf{x})\right]-B P_{i, t-1}(\mathbf{x})\right)\right\} \\
& +\sum_{i=1}^{m} q_{i, t} x_{i} T R_{t-1}\left(\mathbf{x}-\mathbf{e}_{i}\right)+\left(1-\sum_{i=1}^{m} q_{i, t} x_{i}\right) T R_{t-1}(\mathbf{x})
\end{aligned}
$$

where $B P_{i, t-1}(\mathbf{x})=T R_{t-1}(\mathbf{x})-T R_{t-1}\left(\mathbf{x}+\mathbf{e}_{i}\right)$. We define $U C_{i, t}$ by $(6)$ and replace each component of the vector $T C_{t-1}\left(\mathbf{x}+\mathbf{e}_{i}\right)-T C_{t-1}(\mathbf{x})$. This yields a set of equations equivalent to (10), in which the expected cancellation cost is treated simultaneously with the booking request instead of at the time of cancellation

$$
\begin{aligned}
T R_{t}(\mathbf{x}) & =\max _{p}\left\{\sum_{i \in p} d_{i, p, t}\left(f_{i}-U C_{i, t}-B P_{i, t-1}(\mathbf{x})\right)\right\}+\sum_{i=1}^{m} q_{i, t} x_{i} T R_{t-1}\left(\mathbf{x}-\mathbf{e}_{i}\right) \\
& +\left(1-\sum_{i=1}^{m} q_{i, t} x_{i}\right) T R_{t-1}(\mathbf{x})
\end{aligned}
$$

where $B P_{i, t-1}(\mathbf{x})=T R_{t-1}(\mathbf{x})-T R_{t-1}\left(\mathbf{x}+\mathbf{e}_{i}\right)$. The DP model (11) is termed Model D+OB (UC).

Next, we apply the MRT to transform the model into Model I+OB (UC). To achieve that, we need to impose a simplifying assumption on the cancellation rates. We assume an equal cancellation rate $q_{t}$ for all fare classes and use it in the capacity calculation. However, this approximation is not required in $U C_{i, t}$, which can be calculated from class-specific cancellation rates and refund. With this assumption, Model $\mathrm{D}+\mathrm{OB}(\mathrm{UC})$ reduces to

$$
T R_{t}(x)=\max _{p}\left\{\sum_{i \in p} d_{i, p, t}\left(f_{i}-U C_{i, t}-B P_{t-1}(x)\right)\right\}+q_{t} x T R_{t-1}(x-1)+\left(1-q_{t} x\right) T R_{t-1}(x)
$$

where $B P_{t-1}(x)=T R_{t-1}(x)-T R_{t-1}(x+1)$. Note that this equation has the same structure as (8), with $f_{i}$ replaced by $f_{i}-U C_{i, t}$ and the (for a given $x$ and $t$ ) constant term $T N_{t-1}(x)$ replaced by another constant term $q_{t} x T R_{t-1}(x-1)+\left(1-q_{t} x\right) T R_{t-1}(x)$. The effect of considering cancellations is twofold: (i) The cancellation cost may affect which policies are efficient through subtraction of $U C_{i, t}$, and (ii) the entire convex hull will be shifted vertically as result of the cancellations' impact on remaining capacity, resulting in an adjustment of the marginal contributions changing prioritization among flows. However, the optimal solution can still be obtained from an efficient frontier, to which we may apply the MRT directly.

The MRT is carried out on the revenue contribution $f_{i}-U C_{i, t}$, which means that both revenue and expected cancellation cost are transformed. We can create an equivalent efficient frontier with independent demand by setting demand of the virtual policies equal to the marginal demand $M D_{p, t}$ and letting each virtual policy have the marginal contribution $M R_{p, t}-M C_{p, t}$, where the marginal demand and marginal revenue is given by (9), and the marginal cost of opening policy $p$ instead of $p-1$ is defined by

$$
M C_{p, t}=\left(\sum_{i \in p} d_{i, p, t} U C_{i, t}-\sum_{i \in p-1} d_{i, p-1, t} U C_{i, t}\right) /\left(D_{p, t}-D_{p-1, t}\right)
$$

where $p=1, \ldots, k$ denotes the $k$ efficient policies. When including the cost component, the MRT should rightfully be termed the $\mathrm{MCT}$, and we name the resulting fare modifier accordingly: $M R_{p, t}-$ 
$M C_{p, t}=f_{i}-F M_{i, t}^{\text {Contr }}$. Note that mapping back policies to classes is possible only if the policies along the efficient frontier are nested.

This demonstrates equivalence between Model D+OB (TC) and Model I+OB (UC) under the above simplifying assumption on the cancellation rates.

\section{FF Model D+OB (UC)}

Assumptions: Joint overbooking and seat allocation DP model with $n$-dim state variable, demand dependence by fare product, cancellations and overbooking, and refund considered at time of booking. Fare family fare structure.

We consider a fare structure with multiple fare families. For fare families, the fare products within each fare family are unrestricted, which means that customers will purchase the lowest open fare class in each fare family. Therefore, it suffices to consider policies consisting of one fare class (or none) from each fare family.

Formulating Model D+OB (TC) for $n$ fare families and performing the ECT is straightforward, and we can formulate the exact $n$-dim FF Model D+OB (UC) directly

$$
\begin{aligned}
T R_{t}(\mathbf{x}) & =\max _{p}\left\{\sum_{j=1}^{n} D_{j, p, t}\left(f_{j, p}-U C_{j, p, t}-B P_{j, t-1}(\mathbf{x})\right)\right\}+\sum_{j=1}^{n} q_{j, t} x_{j} T R_{t-1}\left(\mathbf{x}-\mathbf{e}_{j}\right) \\
& +\left(1-\sum_{j=1}^{n} q_{j, t} x_{j}\right) T R_{t-1}(\mathbf{x})
\end{aligned}
$$

where $B P_{j, t-1}(\mathbf{x})=T R_{t-1}(\mathbf{x})-T R_{t-1}\left(\mathbf{x}+\mathbf{e}_{j}\right)$.

\section{FF Model I+OB (UC) 1D}

Assumptions: Joint overbooking and seat allocation DP model with 1-dim state variable, demand independence by fare product, cancellations and overbooking, and refund considered at time of booking. Fare family fare structure.

Next, we reduce dimensionality of the DP model to one. We allow for class-specific cancellation rates and refund in $U C_{j, p, t}$, but approximate the cancellation rate in the capacity calculation by an equal cancellation rate $q_{t}$ across all fare families. Because (12) has the same structure as (11), we can apply the MCT directly to get the 1-dim fare family approximate model

$$
\begin{aligned}
T R_{t}(x) & =\sum_{i=1}^{m} M D_{i, t} \times \max \left\{f_{i}-F M_{i, t}^{\text {Contr }}-B P_{t-1}(x), 0\right\} \\
& +q_{t} x T R_{t-1}(x-1)+\left(1-q_{t} x\right) T R_{t-1}(x)
\end{aligned}
$$

which turns out to be equivalent to Model I+OB (UC) in one dimension (considered by Subramanian et al. [1999]), however with transformed demand and fares.

\section{Example: Comparison between MRT and MCT}

To illustrate the difference between the MRT and MCT, we have included an example for the simplest fare structure: A single fare family. The table in Figure 2 is split in two parts for comparison: The left-hand side shows the conventional MRT without cancellations (Fiig et al. [2010]), and the righthand side shows how the concepts are extended to the MCT in the case of cancellations and refund. Demand for each fare class $i$ with associated fare $f_{i}$ is calculated using an exponential sell-up rate as $D_{i}=V_{0} e^{-a\left(f_{i} / f_{0}-1\right)}$, with $V_{0}$ being the base volume and $f_{0}$ the base fare. The cancellation rate for each fare class is calculated as $q_{i}=1-\prod_{\tau=0}^{t}\left(1-q_{i, \tau}\right)$. 
Consider first the MRT. Observe that we obtain $M R_{i}>0$ for the open classes E, M, and $\mathrm{H}$, while $M R_{i}<0$ for the closed classes Q to $\mathrm{T}$. Also note that the fare modifier is constant (except for the $\mathrm{E}$ class [abbreviated $\mathrm{E} / \mathrm{cl}]$ ), which is the case for exponential sell-up when fares are equidistant. In Panel (a), $M R_{i}$ is plotted versus $t$.

Consider next cancellations and refund. The total cost $T C_{i}$ can be calculated by $T C_{i}=c_{i} q_{i} D_{i}$. The resulting marginal contribution $M R_{i}-M C_{i}$ can be compared with $M R_{i}$ to see the impact of cancellations and refund. We observe that the topmost fare is adjusted. Also, $\mathrm{M} / \mathrm{cl}$ becomes inefficient (falls off the convex hull) and is entirely closed. And even though $\mathrm{H} / \mathrm{cl}$ does not have any refund associated with the class (hence $T C_{H}=0$ ), its marginal fare is affected by the overlaying classes. Note that, since $\mathrm{M} / \mathrm{cl}$ is inefficient, $M R_{H}-M C_{H}=\left(\left(T R_{H}-T C_{H}\right)-\left(T R_{E}-T C_{E}\right)\right) /\left(D_{H}-\right.$ $D_{E}$ ). It is also important to note that the cost of cancellation can invert the fares (see Study (iv) in Section 5).

In Panel (b), $M R_{i}-M C_{i}$ is plotted versus $t$. Observe that the curves are no longer parallel. For E/cl, the marginal contribution even increases towards departure. This behavior may seem odd, as it implies that we may potentially accept booking requests closer to departure that we initially rejected (at the same fare amount). We will return to this case in Study (iv) in Section 5.

\begin{tabular}{|c|c|c|c|c|c|c|c|c|c|c|c|}
\hline \multicolumn{6}{|c|}{ Without cancellations } & \multicolumn{6}{|c|}{ With cancellations and refund } \\
\hline$i$ & $f_{i}$ & $D_{i}$ & $T R_{i}$ & $M R_{i}$ & $F M_{i}^{R e v}$ & $c_{i}$ & $q_{i}$ & $T C_{i}$ & $T R_{i}-T C_{i}$ & $M R_{i}-M C_{i}$ & $F M_{i}^{\text {Contr }}$ \\
\hline $\mathrm{E}$ & 2000 & 0.35 & 707 & 2000 & 0 & 1000 & 0.19 & 66 & 641 & 1813 & 187 \\
\hline$M$ & 1800 & 0.40 & 725 & 359 & 1441 & 900 & 0.19 & 68 & 657 & & \\
\hline $\mathrm{H}$ & 1600 & 0.46 & 734 & 159 & 1441 & 0 & 0.19 & 0 & 734 & 881 & 719 \\
\hline$Q$ & 1400 & 0.52 & 731 & -41 & 1441 & 0 & 0.19 & 0 & 731 & -41 & 1441 \\
\hline W & 1200 & 0.59 & 714 & -241 & 1441 & 0 & 0.19 & 0 & 714 & -241 & 1441 \\
\hline $\mathrm{U}$ & 1000 & 0.68 & 677 & -441 & 1441 & 0 & 0.19 & 0 & 677 & -441 & 1441 \\
\hline $\mathrm{K}$ & 800 & 0.77 & 617 & -641 & 1441 & 0 . & 0.19 & 0 & 617 & -641 & 1441 \\
\hline $\mathrm{L}$ & 600 & 0.88 & 527 & -841 & 1441 & 0 & 0.19 & 0 & 527 & -841 & 1441 \\
\hline $\mathrm{T}$ & 400 & 1.00 & 400 & -1041 & 1441 & 0 & 0.19 & 0 & 400 & -1041 & 1441 \\
\hline
\end{tabular}
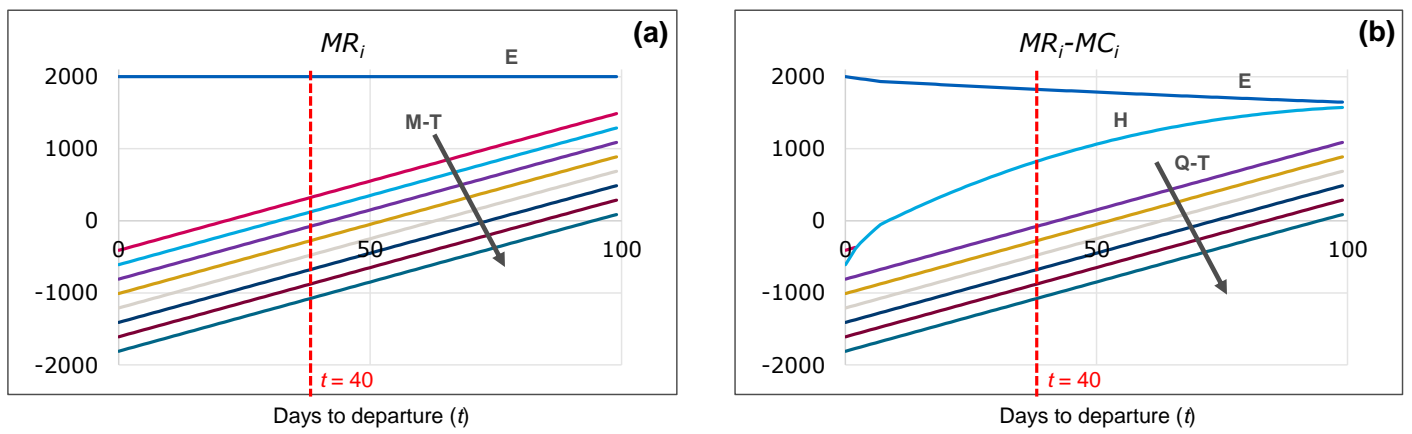

Figure 2: Left: MRT. Right: MCT. The parameters employed correspond to Table 1 Study (ii). In Panels (a) (without cancellations) and (b) (with cancellations and refund), the detailed calculation is illustrated for $t=40$ (vertical dashed line).

\section{Simulation Results}

We will assume the most simple simulation set-up. The airline operates in a monopoly with a single flight leg with capacity $C$. We employ a fare family fare structure consisting of $n=2$ fare families (business $[\mathrm{B}]$ with fare classes $\mathrm{Y}, \mathrm{S}, \mathrm{B}$, and economy $[\mathrm{E}]$ with fare classes $\mathrm{E}, \mathrm{M}, \mathrm{H}, \mathrm{Q}, \mathrm{W}$, $\mathrm{U}, \mathrm{K}, \mathrm{L}, \mathrm{T})$ throughout the study. In the simulator, the RMS and the passenger generation are separated. However, to isolate the performance of the optimization model, we assume that the other 
components of the RMS are perfectly calibrated. The flow diagram of the RMS simulator follows the logic of Figure 3.

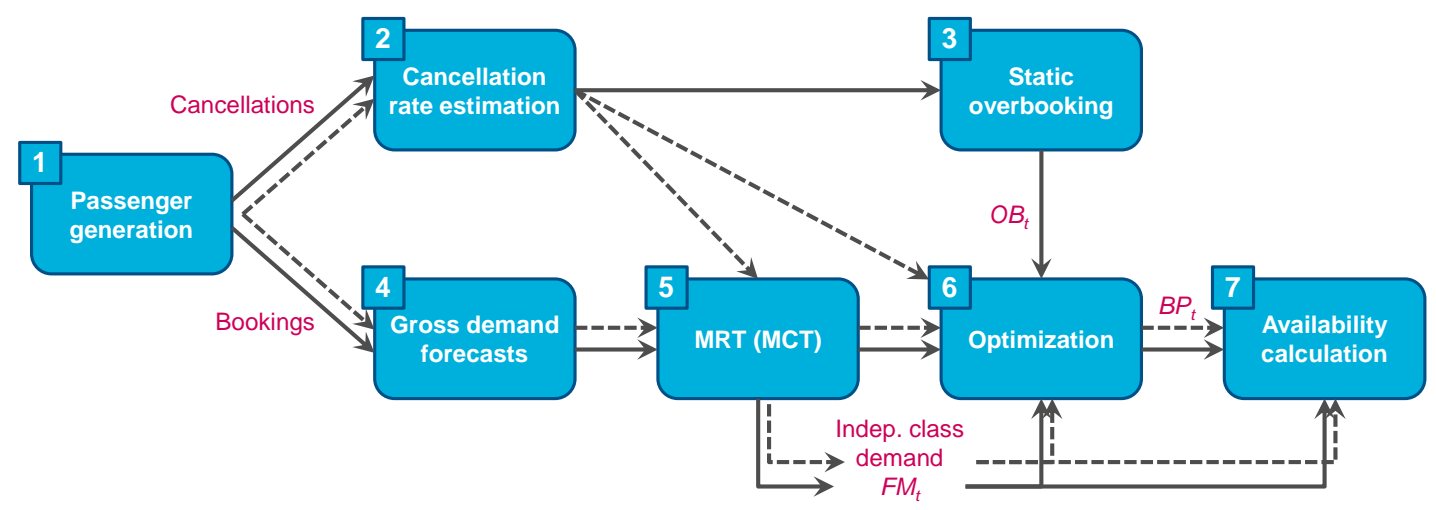

Figure 3: RMS and its components. The 1-dim heuristic model (solid lines) decouple overbooking (box 3) and seat allocation (box 6). The MRT (box 5) calculates independent demand and fare modifiers. The n-dim exact model and 1-dim approximate model (dashed lines) send cancellation rates (box 2) to the MCT (box 5). Cancellation rates are also sent to the optimization model (box 6 ) that performs overbooking and seat allocation jointly, bypassing the overbooking module.

\section{Passenger Generation}

Passenger arrivals (box 1 in Figure 3) are generated according to a Poisson distribution, with a timedependent arrival rate. The decision process of each passenger is simulated according to a choice model, where the passenger selects the fare class with the lowest generalized cost (defined as the fare plus the passenger's disutility cost associated with the restrictions of the fare class), provided that the fare for the fare class is less than the passenger's randomly drawn maximum WTP.

Cancellations are modeled as a Bernoulli process, implicitly assuming that passengers cancel independently of one another and that the probability of cancellation is independent of when the booking was made.

We allow for two passenger types: Business purpose (S) and Leisure purpose (L) passengers. Business purpose passengers exhibit late bookings, high sell-up probability, high sell-across probability, and high cancellation rates. Leisure purpose passengers exhibit early bookings, low sell-up probability, low sell-across probability, and low cancellation rates.

\section{Forecasting}

Fare family forecasting (box 4 in Figure 3) follows the principles from Fiig et al. [2012], which are briefly described here for reference. We assume that the airline offers the policy $\left(f_{B}, f_{E}\right)$, consisting of the lowest open fares in each fare family. We assume the arrival of $V_{0}=V_{0, S}+V_{0, L}$ passengers, where $V_{0, S}$ is the base volume of business purpose passengers and $V_{0, L}$ is the base volume of leisure purpose passengers. The passengers' probability of sell-up from the base fare $f_{0}$ to the offered fare $f_{j}$ in fare family $j$ is expressed as an exponential rate $e^{-a_{u}\left(f_{j} / f_{0}-1\right)}$, and the passengers' product preference is expressed as an exponential sell-across probability $e^{-b_{u}\left(f_{B}-f_{E}\right)}$, where $u$ denotes the passenger type. The demand $D_{j, u}$ for each fare family and passenger type can be expressed as

$$
\begin{aligned}
& D_{B, u}=V_{0, u} e^{-a_{u}\left(f_{B} / f_{0}-1\right)} e^{-b_{u}\left(f_{B}-f_{E}\right)} \\
& D_{E, u}=V_{0, u} e^{-a_{u}\left(f_{E} / f_{0}-1\right)}-D_{B, u}
\end{aligned}
$$


We aggregate over the two passenger types to obtain the total demand $D$ and total revenue $T R$, which are input to the MRT (box 5 in Figure 3)

$$
\begin{aligned}
D & =\sum_{j=B, E} \sum_{u=S, L} D_{j, u} \\
T R & =\sum_{j=B, E} \sum_{u=S, L} D_{j, u} f_{j}
\end{aligned}
$$

The forecast parameters $a_{u}$ and $b_{u}$ are not estimated, but provided as input from the passenger generation (box 1 in Figure 3). This corresponds to a highly accurate and unbiased estimator.

\section{Optimization}

Implementation of the optimization models are described below:

- 1-dim heuristic model (solid lines in Figure 3): Decoupling of overbooking and seat allocation. The implementation of the 1-dim heuristic model uses the static overbooking model of Bodily and Pfeifer [1992], which assumes a single fare class for the cancellation rates, denied boarding compensation, and spoilage cost. For the fare family fare structure, a weighted average is used based on expected business/leisure purpose mix. Seat allocation is performed by Model I, given by (2), with gross capacity $C+O B_{t}$ determined by (1). The decoupling of overbooking and seat allocation means that the overbooking level may not be optimal. Consequently, in practice, RM analysts adjust the overbooking level by an overbooking scaling parameter $\alpha \geq 0$, such that the resulting scaled gross capacity becomes $C+\alpha O B_{t}$. The unscaled (nominal) overbooking level corresponds to $\alpha=1$. We only apply overbooking scaling to the decoupled model.

- $n$-dim exact model (dashed lines in Figure 3): Joint overbooking and seat allocation is performed by FF Model D+OB (UC), given by (12), with physical capacity $C$.

- 1-dim approximate model (dashed lines in Figure 3): Joint overbooking and seat allocation is performed by FF Model I+OB (UC) 1D, given by (13), with physical capacity $C$. As explained in Section 4, class-specific cancellation rates can be included in the acceptance criteria of the DP model, however, only an average (and hence approximate) cancellation rate can be used in the capacity calculation.

\section{Studies}

The parameter settings for the different studies are compiled in Table 1. The first set of studies, (i)-(iii), regards one fare family $(1 \mathrm{FF})$ with one passenger type in order to study the fundamental properties and performance of the 1-dim exact model. The next set of studies, (iv)-(v), considers two fare families $(2 \mathrm{FF})$ with two passenger types, so as to study performance in a more realistic and more complex fare structure:

- Study (i): Sensitivity studies of the 1-dim heuristic and 1-dim exact models (1FF).

- Study (ii): Structural properties of the 1-dim heuristic and 1-dim exact models (1FF).

- Study (iii): Benchmark study of the 1-dim heuristic and 1-dim exact models (1FF).

- Study (iv): Structural properties of the 1-dim heuristic, 2-dim exact, and 1-dim approximate models $(2 \mathrm{FF})$.

- Study (v): Benchmark study of the 1-dim heuristic, 2-dim exact, and 1-dim approximate models $(2 \mathrm{FF})$. 
The focus of the simulation studies is on benchmarking the $n$-dim exact and 1-dim approximate models relative to the 1-dim heuristic model, which serves as our baseline. Exact models produce BPs that account for overbooking, while heuristic models' BPs exclude overbooking. Hence, the BPs cannot be directly compared. A logical and business-relevant comparison is to look instead at class availabilities, which all models produce regardless of their inner mechanics.

For the 1-dim models, we define the availability function by $\operatorname{AVA}_{t}(f)=\operatorname{Argmax}_{x}\left[f \geq B P_{t}(x)\right]$. The 2-dim exact model produces a 2-dim $B P$ vector $B P_{t}(\mathbf{x})=\left(B P_{B, t}(\mathbf{x}), B P_{E, t}(\mathbf{x})\right)$, with the inventory vector $\mathbf{x}=\left(x_{B}, x_{E}\right)$. The class availabilities are calculated analogously to the 1-dim model by $\operatorname{AVA}_{t}\left(f_{j}\right)=\operatorname{Argmax}_{x_{j}}\left[f_{j} \geq B P_{j, t}(\mathbf{x})\right]$ for fare family.

The following caveats should be noted with respect to the simulation results. They are specific to the set of network, competitive environments, fare structure, and model assumptions of these particular studies. While indicative, they may change under other input conditions.

\section{(i) Sensitivity Studies of the 1-dim Heuristic and 1-dim Exact Models}

In this set of studies, we investigate how the overbooking level from the 1-dim exact model is affected by WTP, demand level, and refund.

In Figure 4, we display the overbooking level as a function of $t$. The overbooking level can be identified as the availability of the highest fare class $(\mathrm{E} / \mathrm{cl})$. We observe that the overbooking level for the 1-dim exact model is quite sensitive to the selected parameters. In contrast, for the 1-dim heuristic model (black solid line), the overbooking level is independent of WTP, demand level, and refund; in the figure, the overbooking level is unaffected by the different parameters.
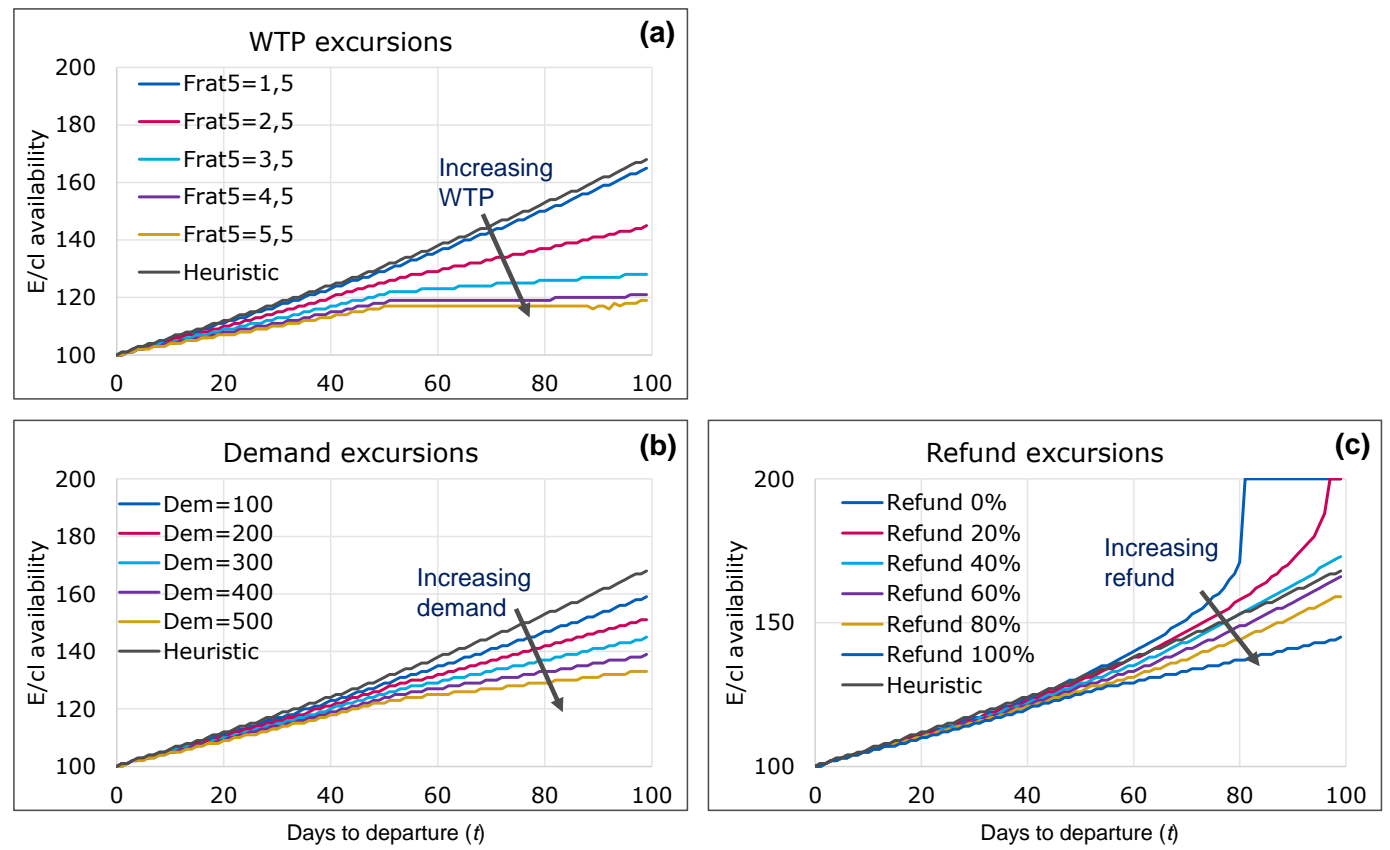

Figure 4: Sensitivity studies of the 1-dim exact model, displaying $\mathrm{E} / \mathrm{cl}$ availability versus $t . \mathrm{E} / \mathrm{cl}$ represents the highest fare class. For parameters, see Table 1 (i), cases (a)-(c).

Panel (a) shows that the overbooking level decreases with increased WTP. This can be understood from the fact that revenue is comprised of two components: Volume (overbooking) and mix (seat allocation). As WTP increases, reducing the overbooking level (lower load factor [LF]) prevents buy-down to lower classes (improved mix). 
Panel (b) illustrates the overbooking level as function of demand. We observe that the overbooking level decreases as demand increases. There are two aspects to this. First, higher demand presents more opportunities to sell. Hence, we can reduce the risk of DBs by lowering the overbooking level. Second, with higher demand, we can improve the class mix by lowering the overbooking level, thereby forcing passengers to buy up.

Finally, in Panel (c), we observe that the overbooking level decreases as refund increases. The refund level varies from non-refundable (0 per cent) to fully refundable (100 per cent). The 1-dim heuristic model is traditionally derived assuming 100 per cent refund, thereby providing the most conservative overbooking level. With lower refund levels, it pays off to overbook more, because the airline obtains a secondary revenue stream from passengers who cancel. For the airline, these passengers do not occupy capacity, and the airline may resell the seat. Overbooking diverges specifically for 0 per cent refund, since, with the selected parameters, revenue from non-refundable passengers who cancel outweighs the cost of compensation for DBs. This may seem fictitious, but it is included here for illustration purposes. It results from the lack of long tails in the binomial cancellation distribution, which provides a too optimistic view of the risk of overselling. In the real world, the airline would put a cap on the overbooking level.

\section{(ii) Structural Properties of the 1-dim Heuristic and 1-dim Exact Models}

In this set of studies, we compare the structural properties of the 1-dim exact and 1-dim heuristic models.

Figure 5 displays availabilities for $\mathrm{E} / \mathrm{cl}-\mathrm{T} / \mathrm{cl}$. The parameters are modified with respect to those of Figure 4 to align more closely with the real world. Hence, refund is differentiated among fare classes, providing partial refundability only to the highest classes. Passengers' price sensitivity displays a linear dependence on $t$. Finally, the cancellation rates are assumed to be higher in the last week before departure to better model actual passenger behavior.

Note that, in Figure 5, availability for both the heuristic and exact models is not monotonous with respect to $t$. This type of behavior is absent in the pure seat allocation models (see e.g. Lee and Hersh [1993] or Lautenbacher and Stidham [1999]), but is a result of overbooking. Passengers separated in $t$ have different cancellation rates, resulting in different cost of cancellation and, hence, availability.

Comparing the 1-dim heuristic and 1-dim exact models, we see important structural differences. $\mathrm{M} / \mathrm{cl}$ is open in the 1-dim heuristic model, but is closed for all $t$ in the 1-dim exact model because refundability makes it inefficient (see also Figure 2 Panel (b)). Another striking difference is that $\mathrm{H} / \mathrm{cl}$ is open longer in the 1-dim exact model (closes at $t=9$ ) than in the 1-dim heuristic model (closes at $t=31$ ). Also, $\mathrm{L} / \mathrm{cl}$ opens early in the 1-dim heuristic model, but is entirely closed in the 1-dim exact model.

\section{(iii) Benchmark Study of the 1-dim Heuristic and 1-dim Exact Models}

The purpose of this study is to evaluate the impact on revenue and DB levels of applying the 1-dim exact model relative to our 1-dim heuristic baseline model. The parameters are identical to the previous study and correspond to realistic settings.

In Figure 6, Panel (a) illustrates the revenue index (normalization with the revenue of the unscaled heuristic model) versus LF for both the 1-dim exact and 1-dim heuristic models, applying different overbooking scaling parameters. The overbooking scaling parameters vary from $\alpha=0.5$ to $\alpha=1.25$.

The unscaled heuristic model has a revenue index of 100. Note that the revenue of the 1-dim heuristic model can be improved by reducing the nominal overbooking level. Revenue maximization occurs for a scaling of $\alpha=0.75$. The corresponding revenue gain is 1.67 per cent. However, this gain will be difficult to replicate for an airline in practice, since the scaling parameter in general is scenario-specific. Reducing the overbooking level also affects the LF, which decreases by 3.64 pp. 

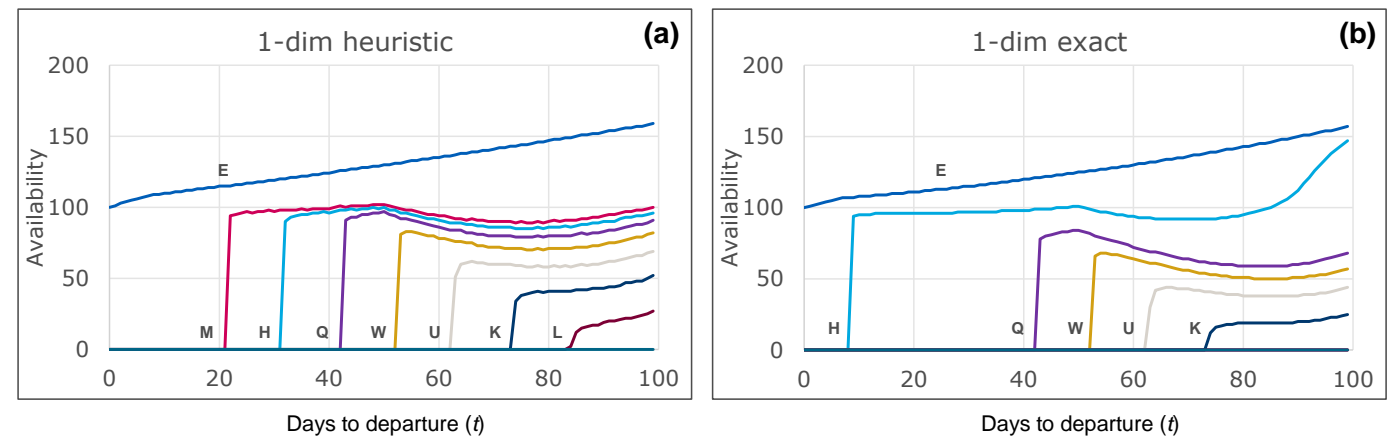

Figure 5: Comparison of the structural properties of the 1-dim heuristic and 1-dim exact models for the one fare family fare structure. The graphs are labelled by the respective fare classes. For parameters, see Table 1 (ii).

The 1-dim exact model produces a revenue gain of 3.25 per cent, which is a significant improvement over the 1-dim heuristic model without scaling. Also note that the optimal revenue point occurs at the same LF and DB level as the revenue-maximized heuristic model.

The improvement in revenue cannot be explained by a reduced cost of DB (this is captured in the revenue gain of the 1-dim scaled heuristic model, which has similar DB level but lower revenue as displayed in Panel (b)). To understand this, we in Panel (c) compare the fare class mix of the 1-dim exact model and the 1-dim scaled heuristic model. Observe that the 1-dim heuristic model is too open by allowing bookings in $\mathrm{M} / \mathrm{cl}$ and $\mathrm{L} / \mathrm{cl}$, whereas the 1 -dim exact model is closed. At the same time, the 1-dim heuristic model is too aggressive by closing $\mathrm{H} / \mathrm{cl}$ too early, since it does not account for the partial refundability of $\mathrm{M} / \mathrm{cl}$.

The incremental gain can be explained from the fact that the 1-dim exact model considers cancellation cost in its seat allocation, while also accounting for the interrelationship between overbooking and fare class mix - both of which are ignored in the 1-dim heuristic model.

\section{(iv) Structural Properties of the 1-dim Heuristic, 2-dim Exact, and 1-dim Approximate Models}

In this study, we add the business fare family to the fare structure, where the highest class, $\mathrm{Y} / \mathrm{cl}$, is fully refundable, while the next two classes, $\mathrm{S} / \mathrm{cl}$ and $\mathrm{B} / \mathrm{cl}$, are partially refundable.

Figure 7 displays the availabilities of all fare classes across the booking horizon of the 1-dim heuristic model in Panel (a), the 2-dim exact model in Panel (b), and the 1-dim approximate model in Panel (c). Comparing the 1-dim heuristic model with the 2-dim exact model, there are noteworthy structural differences. The $\mathrm{Y} / \mathrm{cl}$ and $\mathrm{S} / \mathrm{cl}$ are inverted far from departure. The 1-dim heuristic model incorrectly assumes that $\mathrm{Y} / \mathrm{cl}$ is more profitable than $\mathrm{S} / \mathrm{cl}$ by incorrectly assuming that all classes are fully refundable. As we approach departure, however, the cost of cancellation decreases, thereby recovering the sales order. Also, observe that the 2-dim exact model is more closed than the 1-dim heuristic model, which is most apparent by comparing the availabilities of the lower classes.

Comparing the 2-dim exact model to the 1-dim approximate model, we observe similar structural properties, although the 1-dim approximate model is compressed (business availability is lower, while economy availability is higher). This is because the 1-dim approximate model employs an average cancellation rate that falls in between the business and economy cancellation rates, in essence underestimating cancellation rates for business class while overestimating cancellation rates for economy class. 


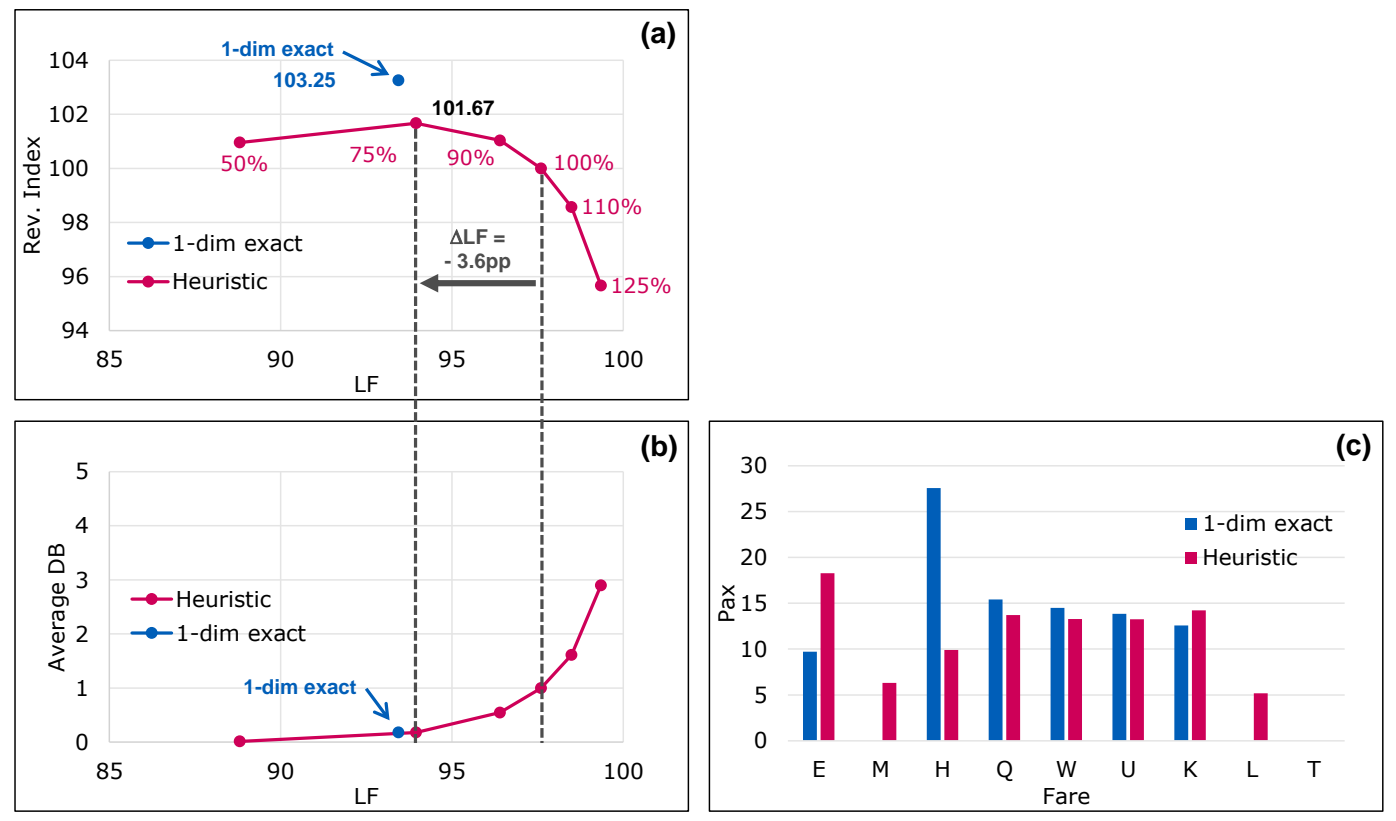

Figure 6: Benchmark study for one fare family comparing the overbooking scaled heuristic and exact models. Simulation parameters correspond to those of Figure 5 and are displayed in Table 1 (iii). Revenue index (a) and DBs (b) versus LF are shown for the models. In (c), fare class mix is compared between the models at LF equal to 94 per cent.

\section{(v) Benchmark Study of the 1-dim Heuristic, 2-dim Exact, and 1-dim Approximate Models}

This study evaluates the revenue impact and DB level of the three models. The fare structure and parameters are identical to the previous study.

Panel (a) in Figure 8 illustrates the revenue index versus LF for the 1-dim heuristic, 2-dim exact, and 1-dim approximate models, applying different overbooking scaling parameters. Note that revenue maximization for the 1-dim heuristic model occurs with a reduced overbooking level $\alpha \sim 0.75$, providing a revenue gain of 0.42 per cent. Again, the impact of reduced overbooking can be observed as a reduced LF, which decreases $1.4 \mathrm{pp}$ (Panel (a)), and fewer DBs, which decrease from 26.6 DB/10,000 flown to 6.4 DB/10,000 flown (Panel (b)). Both the 2-dim exact and 1-dim approximate models produce a revenue gain of about 1.25 per cent, which is again better than the 1-dim scaled heuristic model.

To further compare the performance of the three models, Panel (c) compares the fare class mix of all models at the same LF of approximately 96 per cent (revenue maximizing for the 1-dim heuristic model). The 1-dim heuristic model is too open, allowing bookings in $\mathrm{L} / \mathrm{cl}$, where the other models are closed. The 1-dim heuristic model is also too open in $\mathrm{Y} / \mathrm{cl}$. All observations are consistent with the structural properties discussed previously.

The revenue gains from the 2-dim exact and 1-dim approximate models are lower than in Study (iii) because bookings occur much closer to departure when cancellation rates are lower, particularly among business purpose passengers.

Comparing the 2-dim exact and the 1-dim approximate models, we observe a near-identical performance in terms of revenue, LF, DB, and fare class mix. In summary, the 2-dim exact model and its 1-dim approximation are both able to balance revenue from overbooking and revenue from seat allocation at the same time. This is important, since the 1-dim approximate model can easily 

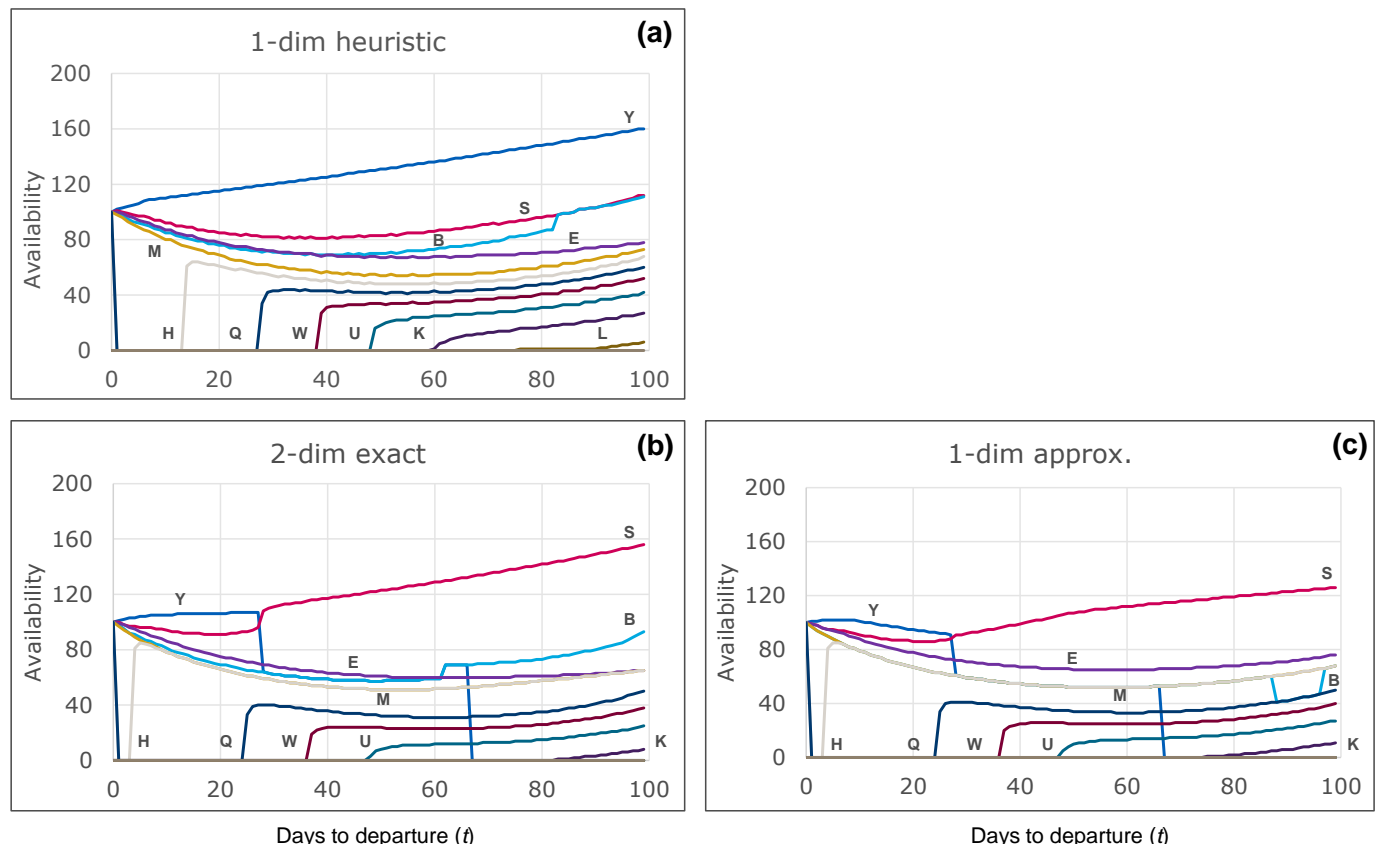

Figure 7: Fare class availabilities versus $t$ for the 1-dim heuristic (a), 2-dim exact (b), and 1-dim approximate (c) models. Calculations are performed for the fare structure with two fare families. For parameters, see Table 1 (iv).

be implemented in commercial RMS.

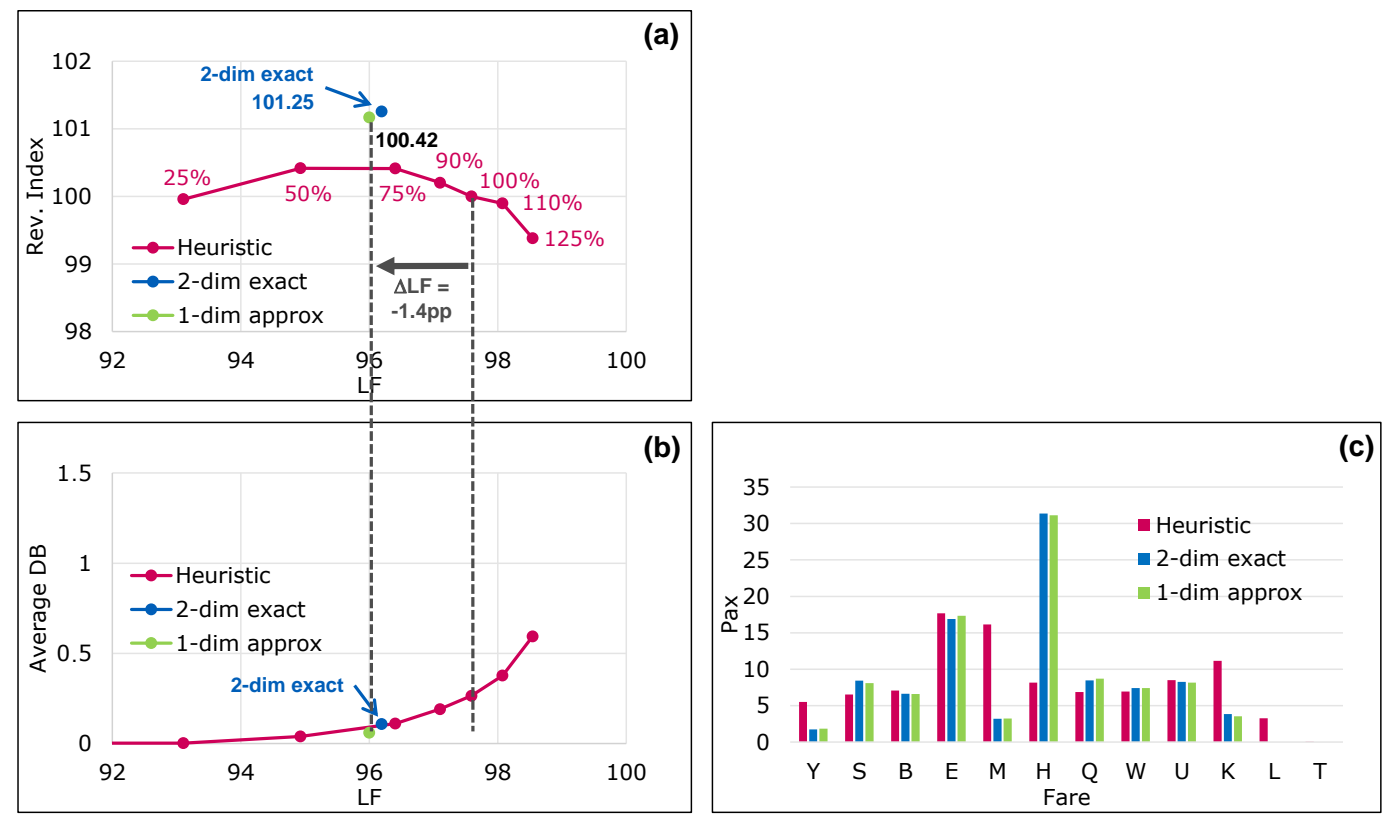

Figure 8: Benchmark study for two fare families comparing the 1-dim scaled heuristic, 2-dim exact, and 1-dim approximate models. For parameters, see Table 1 (v). 


\begin{tabular}{|c|c|c|c|c|c|}
\hline Studies & (i)-a & (i)-b & (i)-c & (ii), (iii) & (iv), (v) \\
\hline \multicolumn{6}{|l|}{ Total demand } \\
\hline Total demand & 300 & 100 to 500 & 300 & 300 & 150 to 300 \\
\hline \multicolumn{6}{|l|}{ Refundability } \\
\hline Economy class & $100 \%$ & $100 \%$ & $0 \%$ to $100 \%$ & \multicolumn{2}{|c|}{$\mathrm{EM} / 50 \% \mathrm{HQWUKLT} / 0 \%$} \\
\hline Business class & & - & & & $\mathrm{Y} / 100 \% \mathrm{SB} / 50 \%$ \\
\hline \multicolumn{6}{|l|}{ Leisure purpose passenger } \\
\hline$q_{t}$ last week before $t=0$ & $0.5 \%$ & $0.5 \%$ & $0.5 \%$ & $1.0 \%$ & $0.5 \%$ \\
\hline$q_{t}$ otherwise & $0.5 \%$ & $0.5 \%$ & $0.5 \%$ & $0.4 \%$ & $0.2 \%$ \\
\hline Build-up (cumulated bookings) & \multicolumn{4}{|c|}{$t<50: 1-0.004 t ; t>50: 1.6(1-0.01 t)$} & $1-0.01 t$ \\
\hline Sell-up: Frat5 & 1.5 to 5.5 & 2.5 & 2.5 & $5-0.033 t$ & $3-0.013 t$ \\
\hline Sell-across: $\operatorname{Ln}(2) / b$ & & - & - & & 100 \\
\hline \multicolumn{6}{|l|}{ Business purpose passenger } \\
\hline$q_{t}$ last week before $t=0$ & - & - & - & - & $1 \%$ \\
\hline$q_{t}$ otherwise & - & - & - & - & $0.4 \%$ \\
\hline Business/Leisure purpose mix & - & - & - & - & $0.8(1-0.01 t)$ \\
\hline Sell-up: Frat5 & - & - & - & - & 5 \\
\hline Sell-across: $\operatorname{Ln}(2) / b$ & - & - & - & - & 346 \\
\hline
\end{tabular}

Table 1: Parameter settings for Studies (i) $-(\mathrm{v})$. Flight capacity $C=100$. Fare family fare classes and fares: Business: Y/2600, S/2400, B/1100; Economy: E/2200, M/1800, H/1600, Q/1400, W/1200, $\mathrm{U} / 1000, \mathrm{~K} / 800, \mathrm{~L} / 600, \mathrm{~T} / 400$. Parametrization of booking horizon: $t=[0 ; 100]$ with departure at $t=0$. The price elasticity parameter $a$ is expressed in terms of the Frat5. DB compensation: $D B C$ equal to 1000 plus reissue of the ticket (highest fare in the fare family). For further details on the implementation of sell-up, sell-across, build-up, see Fiig et al. [2012]

\section{Conclusion}

Our paper considers the general $m$-dim DP model of joint overbooking and seat allocation that includes customer choice behavior in a general fare structure (see Hjorth [2013] and Sierag et al. [2015]). We have applied this model to fare families, which, because of the prevalence in the airline industry, is of great commercial interest. We demonstrate that the exact model and its 1-dim approximation overcome several of the inadequacies of the heuristic approach (currently applied in the industry) by correctly accounting for the influence of demand level, refund, class-specific cancellation rates, and WTP.

Generally, we have seen that the 1-dim heuristic model is too open both for high and low fare classes. For high fare classes, this is a result of ignoring cancellation cost of refundable fare products, while for low classes it is a result of ignoring that overbooking will impact yield negatively.

We demonstrate that our 1-dim DP model not only delivers near-optimal performance (compared to the exact model) in terms of revenue, LF, and fare class mix, but also provides significant revenue gains of 1 to 3 per cent. At the same time, importantly, the DB level is reduced from $26.6 \mathrm{DB} / 10,000$ flown in the heuristic approach to $6.4 \mathrm{DB} / 10,000$ flown in both the exact model and its 1-dim approximation.

While the present work demonstrates great potential for improving the performance, we have so far ignored the impact of both networks and competitive effects. This is naturally a topic for future research.

\section{Acknowledgement}

We would like to thank Sara Skytte Olsen for her invaluable contributions to this research. 


\section{References}

J. Subramanian, S. Stidham, and C. J. Lautenbacher. Airline yield management with overbooking, cancellations, and no-shows. Transportation Science, 33(2):147-167, 1999.

T. Fiig, K. Isler, C. Hopperstad, and P. P. Belobaba. Optimization of mixed fare structure: Theory and applications. Journal of Revenue and Pricing Management, 9(1):152-170, 2010.

B. C. Smith, J. F. Leimkuhler, and R. M. Darrow. Yield management at american airlines. Interfaces, $22: 8-31,1992$.

T. Gorin, W. G. Brunger, and M. M. White. No-show forecasting: A blended cost-based, pnradjusted approach. Journal of Revenue and Pricing Management, 5(3):188-206, 2006.

P. LeBeau. United will now offer up to $\$ 10,000$ for passengers who give up their seat on an overbooked flight. Online: https://www.cnbc.com/2017/04/27/united-will-now-offer-up-to-10000-forpassengers-who-give-up-their-seat-on-an-overbooked-flight.html, April 2017.

M. Rothstein and A. W. Stone. Passenger booking levels. Proceedings 7th AGIFORS Symposium, American Airlines, New York, 1967.

R. Hjorth. Overbooking in a fenceless fare structure. Master's thesis, Department of Management Engineering, Technial University of Denmark, 2013.

D. D. Sierag, G. M. Koole, R. D. van der Mei, J. I. van der Rest, and B. Zwart. Revenue management under customer choice behaviour with cancellations and overbooking. European Journal of Operational Research, 246(1):170-185, 2015.

M. Rothstein. Or forum - or and the airline overbooking problem. Operations Research, 33(2): 237-248, 1985.

R. M. Ratliff. Ideas on overbooking. Presentation at AGIFORS Reservation and Yield Management Study Group Meeting, Melbourne, Australia, 1998.

K. Littlewood. Forecasting and control of passenger bookings. Journal of Revenue and Pricing Management, 4(2):111-123, 2005. Presentation given at the 12th AGIFORS Symposium in October 1972.

P. P. Belobaba. Or practice - application of a probabilistic decision model to airline seat inventory control. Operations Research, 37(2):183-197, 1989.

T. C. Lee and M. Hersh. A model for dynamic airline seat inventory control with multiple seat bookings. Transportation Science, 27(3):252-265, 1993.

C. J. Lautenbacher and S. Stidham. The underlying markov decision process in the single-leg airline yield-management problem. Transportation Science, 33(2):136-146, 1999.

W. L. Cooper, T. Homem de Mello, and A. J. Kleywegt. Models of the spiral-down effect in revenue management. Operations Research, 54(5):968-987, 2006.

K. Talluri and G. van Ryzin. Revenue management under a general discrete choice model of consumer behavior. Management Science, 50(1):15-33, 2004a.

K. Talluri and G. van Ryzin. The Theory and Practice of Revenue Management. Springer, 2004b.

E. Shlifer and Y. Vardi. An airline overbooking policy. Transportation Science, 9(2):101-114, 1975.

S. E. Bodily and P. E. Pfeifer. Overbooking decision rules. Omega, 20(1):129-133, 1992. 
R. Klophaus and S. Pölt. Airline overbooking with dynamic spoilage costs. Journal of Revenue and Pricing Management, 6(1):9-18, 2007.

M. Rothstein. An airline overbooking model. Transportation Science, 5(2):180-192, 1971.

W. Wang and D. Walczak. Overbooking under dynamic and static policies. Journal of Revenue and Pricing Management, 15(6):534-553, 2016.

J. Alstrup and S. Boas. Bookingpolitik for fly med to passagertyper. Master's thesis, Technical University of Denmark, 1984.

T. Fiig, K. Isler, C. Hopperstad, and S. Skytte Olsen. Forecasting and optimization of fare families. Journal of Revenue and Pricing Management, 11(3):322-342, 2012.

R. Hjorth, T. Fiig, N. Bondoux, and J. Larsen. Joint overbooking and seat allocation for fare families. Technical report, 2016. 